\title{
Lipopolysaccharide/adenosine triphosphate induces IL-1ß and IL-18 secretion through the NLRP3 inflammasome in RAW264.7 murine macrophage cells
}

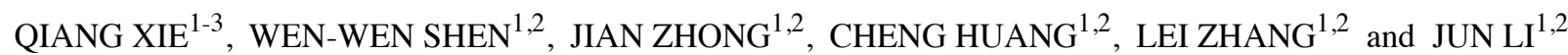 \\ ${ }^{1}$ School of Pharmacy, Anhui Medical University; ${ }^{2}$ The Key Laboratory of Anti-inflammatory and \\ Immune Medicine (Anhui Medical University), Ministry of Education, Hefei, Anhui 230032; \\ ${ }^{3}$ PET/CT Center, Anhui Provincial Hospital, Hefei, Anhui 230001, P.R. China
}

Received December 3, 2013; Accepted March 28, 2014

DOI: $10.3892 /$ ijmm.2014.1755

\begin{abstract}
The NOD-like receptor family, pyrin domaincontaining 3 (NLRP3) inflammasome plays pivotal roles in inflammation and autoimmunity. The NLRP3 inflammasome is activated in response to various signals, including pathogenassociated molecular patterns (PAMPs) and danger-associated molecular patterns (DAMPs). However, its role in inflammation remains unclear. In this study, we used lipopolysaccharide (LPS) and adenosine triphosphate (ATP) to simulate an inflammatory environment as the testing model. We found that the exposure of RAW264.7 cells to LPS/ATP triggered the activation of caspase-1 $(\mathrm{P}<0.01)$ and the cleavage of interleukin (IL) $1 \beta(P<0.01)$, as well as the release of other cytokines, such as IL-18 $(\mathrm{P}<0.01)$ and IL-33 $(\mathrm{P}<0.01)$. Extracellular potassium chloride at a high concentration $(150 \mathrm{mM})$ abrogated the secretion of IL-1 $\beta$ and IL-18 $(\mathrm{P}<0.01)$, but did not reduce the processing of IL-33 ( $>00.05)$. In addition, the silencing of NLRP3 with small interfering RNA (siRNA) suppressed the generation of proinflammatory cytokines, such as IL-1 $\beta$ $(\mathrm{P}<0.01)$, IL-18 $(\mathrm{P}<0.01)$, but not IL-33 $(\mathrm{P}>0.05)$, along with the decreased mRNA and protein expression of NLRP3 and caspase-1 $(\mathrm{P}<0.05)$. However, extracellular potassium at a high concentration and NLRP3 siRNA did not affect the level of apoptosis-associated speck-like protein containing a caspase recruitment domain (CARD) (ASC; $\mathrm{P}>0.05$ ). Our results suggest that the NLRP3/ASC/caspase-1 axis participates in the regulation of pro-imflammatory cytokine secretion in RAW264.7 cells, particularly the generation of IL-1 $\beta$ and IL-18.
\end{abstract}

Correspondence to: Professor Jun Li, School of Pharmacy, Anhui Medical University, 81 Meishan Road, Hefei, Anhui 230032, P.R. China E-mail: 1j@ahmu.edu.cn

Key words: NOD-like receptor family, pyrin domain-containing 3, caspase-1, inflammasome, interleukin-1 $\beta$, interleukin-18, RAW264.7 cells

\section{Introduction}

Innate immunity offers the first defense in mammals, depending on its capacity to rapidly detect invading pathogens and then eliminating the 'foreign danger' (1). However, innate immunity is also involved in the development of inflammatory responses that occur in a sterile milieu (2). Inflammasomes are intracellular multiprotein complexes that regulate the activity of caspase-1 and can be activated by various cellular dangers that trigger the processing and release of pro-inflammatory cytokines to engage innate immunity (3). Nucleotide-binding oligomerization domain (NOD)-like receptors (NLRs) have recently been identified as innate immune receptors that play a pivotal role in initiating the inflammatory response (4). The NLR family, pyrin domain-containing 3 (NLRP3) inflammasome is currently one of the most fully characterized inflammasomes. The NLRP3 inflammasome consists of the NLRP3 scaffold, the apoptosisassociated speck-like protein containing a caspase recruitment domain (CARD) (ASC) adaptor and caspase-1 $(5,6)$. The NLRP3 inflammasome is activated in response to a variety of signals that include pathogen-associated molecular patterns (PAMPs) or danger-associated molecular patterns (DAMPs) (6). The NLRP3 inflammasome is a multiprotein complex that activates cysteine protease caspase- 1 , which leads to the maturation and secretion of pro-inflammatory cytokines, such as interleukin-1 $\beta$ (IL)-1 $\beta$ and IL-18 (7).

The molecular mechanisms of NLRP3 inflammasome assembly and activation have been gradually explored. Previous studies have indicated that reactive oxygen species (ROS) generation, potassium $\left(\mathrm{K}^{+}\right)$efflux, and the release of cathensin $\mathrm{B}$ can activate the NLRP3 inflammasome (3). It is also well recognized that the activation of the NLRP3 inflammasome requires double signals: one is a priming signal from PAMPs that controls the expression of NLRP3; the second stimulus is DAMPs that induce the activation of caspase-1 $(3,5,8,9)$. Lipopolysaccharide (LPS), the major outer membrane component in Gram-negative bacteria, is one of the most characterized PAMPs (10). Adenosine triphosphate (ATP), released from dying cells, has a high concentration within the cell. Extracellular ATP, one of the DAMPs, serves as the second signal for NLRP3 inflammasome activation (11). The IL-1 family has been recognized to play important roles in 
inflammation with pro-inflammatory properties, associated with acute and chronic inflammation, and plays a significant role in the innate immune defense (12). There are several cytokines in the IL-1 family, including IL-1 $\alpha$, IL-1 $\beta$, IL-18 and IL-33. Among the IL-1 family of cytokines, IL-1 $\beta$ is the most characterized cytokine; studies have demonstrated that IL-1 $\beta$ plays an essential role in the pathophysiology of autoimmune diseases $(13,14)$. Importantly, IL-1 $\beta$ maturation requires 2 steps: first, the upregulation of the precursor of IL-1 $\beta$ (pro IL-1 $\beta$ ) transcription through Toll-like receptor (TLR) ligands, such as LPS; and second, the activation of the cysteine protease, caspase-1 (15). Caspase- 1 is a proteolytic enzyme that processes the inactive precursor of IL-1 $\beta$ into the mature form, and is termed IL- $1 \beta$ converting enzyme (ICE). Caspase-1 itself acts as an inactive precursor in the cytoplasm and can be activated by NLRP3 inflammasome assembly and subsequent proteolytic self-processing (16).

It has been widely accepted that the NLRP3 inflammasome plays critical roles in inflammation and immune system regulation. However, whether LPS and/or ATP affect the regulation of NLRP3 inflammasome components, the induction of caspase-1, the cleavage and release of IL-1 $\beta$, IL-18 and IL-33 remains unclear. Thus, in this study, we performed a series of experiments using RAW264.7 murine macrophage cells. Our findings suggested that a high concentration of potassium chloride or the silencing of NLRP3 with small interfering (siRNA) partially abrogated the LPS-induced secretion of pro-inflammatory cytokines in vitro.

\section{Materials and methods}

Chemicals and reagents. Dimethyl sulfoxide (DMSO), ATP, ultrapure LPS and potassium chloride were purchased from Sigma (St. Louis, MO, USA). Phenylmethanesulfonyl fluoride (PMSF) and RIPA lysis buffer were both from the Beyotime Institute of Biotechnology (Shanghai, China). Rabbit antiNLRP3 polyclonal antibody, rabbit anti-ASC polyclonal antibody, rabbit anti-caspase-1 polyclonal antibody, mouse anti$\beta$-actin monoclonal antibody were all purchased from Santa Cruz Biotechnology, Inc. (Santa Cruz CA, USA). Dulbecco's modified Eagle's medium (DMEM) and fetal bovine serum (FBS) were obtained from HyClone Laboratories, Inc. (Logan, UT, USA) and Tianchang Heng Sheng Medical Devices Co., Ltd. (Huzhou, China), respectively. The mouse IL-1 $\beta$, IL-18 and IL-33 enzyme-linked immunosorbent assay (ELISA) kits were purchased from R\&D Systems (Minneapolis, MN, USA). Small interfering RNA targeting NLRP3 were purchased from GenePharma Corp. (Shanghai, China). All other chemicals were of reagent grade.

Cell culture and treatment. The RAW264.7 murine macrophages cells were obtained from the American Type Culture Collection (ATCC; Rockville, MD, USA). The cells were cultured in high glucose DMEM medium (HyClone Laboratories, Inc.) supplemented with $10 \%$ (v/v) heat-inactivated FBS (Tianchang Heng Sheng Medical Devices Co., Ltd.), 100 U/ml penicillin and $100 \mathrm{mg} / \mathrm{ml}$ streptomycin (both from Beyotime Institute of Biotechnology) and kept in a $37^{\circ} \mathrm{C}$ incubator under a humidified atmosphere containing $5 \% \mathrm{CO}_{2}$. The cells were allowed to adhere overnight $\left(37^{\circ} \mathrm{C}, 5 \% \mathrm{CO}_{2}\right)$ and washed with fresh medium to remove unattached cells prior to the experiment. Our experi- ment on RAW264.7 cells included 2 groups. In the first group, cells were treated with LPS, ATP and a high concentration of $\mathrm{K}^{+}$, including 8 subgroups. RAW264.7 cells were stimulated with LPS $(200 \mathrm{ng} / \mathrm{ml})$ for $6 \mathrm{~h}$ and $2 \mathrm{mM}$ ATP for an additional $30 \mathrm{~min}$ (LPS + ATP) or ATP alone. Potassium chloride $(150 \mathrm{mM})$ was additionally added to the cell culture medium for $30 \mathrm{~min}$ prior to LPS and/or ATP stimulation in the last 4 subgroups. In the second group, the cells were treated with LPS, ATP and NLRP3 siRNA, including 7 subgroups. RAW264.7 cells without treatment (untreated group) were used as the controls. In the second group, RAW264.7 cells were stimulated with LPS and/or ATP as indicated in the first group. We transfected the RAW264.7 cells with NLRP3 siRNA prior to stimulation with LPS and/ or ATP in the last 3 subgroups. After these treatments, supernatants were collected for ELISA assay. The cells were washed with PBS 3 times, and then the cell lysis (the mixture of RIPA and PMSF, v/v=100:1) was added into the culture plate without any culture medium. The whole progress of protein extraction was carried out on ice. The mixture, including cell lysis and the cells was sucked out and then place into a $1.5 \mathrm{ml}$ EP tube. The protein sample of RAW264.7 cells for western blot analysis was obtained by vortexing the mixture evenly and centrifugation at $12,000 \mathrm{rpm}$, at $4^{\circ} \mathrm{C}$ for $40 \mathrm{~min}$, then removing the supernatant and adding protein loading buffer.

ELISA. The RAW264.7 cells ( $2 \times 10^{6}$ cells/well) were seeded in a 6-well-plate, and the cells are treated as indicated. IL-1 $\beta$, IL-18 and IL-33 levels in the cell supernatants were measured using ELISA kits according to the manufacturer's instructions.

Quantitative reverse transcription-polymerase chain reaction (qRT-PCR). Total RNA was extracted from the RAW264.7 cells using TRIzol reagent (Invitrogen, Carlsband, CA, USA). cDNA was obtained using a ThermoScript RT-PCR synthesis kit (Fermentas, San Diego, CA, USA) according to the manufacturer's instructions. The sequences of primers used fro qRT-PCR are listed in Table I. qRT-PCR analyses for the mRNA expression of NLRP3, ASC, caspase-1 and $\beta$-actin were performed using QuantiFast SYBR-Green RT-PCR kits (Qiagen, Hilden, Germany) and analyzed using the PikoReal 96 Real-Time PCR system (Thermo Scientific, Vantaa, Finland). The mRNA level of $\beta$-actin was measured as an internal control. Data were computed using the PikoReal 96 Real-Time PCR system software. Three experiments from 3 independent RNA samples were performed.

Western blot analysis. The RAW264.7 cells were collected and lysed in lysis buffer (Beyotime Institute of Biotechnology), then the whole cell lysate was separated by SDS-PAGE and further transfered onto PVDF membranes (Millipore Corp., Billerica, MA, USA). The membranes were then incubated at room temperature for $3 \mathrm{~h}$ with $5 \%$ non-fat milk in Tris-buffered saline (TBS) solution with the detergent, Tween-20 (TBST), and were subsequently incubated with specific primary antibody at $4^{\circ} \mathrm{C}$ for $12 \mathrm{~h}$ in blocking solution. Specific primary antibodies targeting NLRP3, ASC, caspase- 1 and $\beta$-actin were used 1:1,000, 1:500, 1:500, 1:500, respectively. Following 3 washes with TBST, the membranes were incubated at room temperature for $1 \mathrm{~h}$ with HRP-conjugated secondary antibody (anti-rabbit and anti-mouse, respectively). The protein 
Table I. Primers used for qRT-PCR.

\begin{tabular}{lll}
\hline Gene & \multicolumn{1}{c}{ Forward primer } & \multicolumn{1}{c}{ Reverse primer } \\
\hline NLRP3 & 5'-CCTGACCCAAACCCACCAGT-3' & 5'-TTCTTTCGGATGAGGCTGCTTA-3' \\
ASC & 5'-TGAGCAGCTGCAAACGACTA-3' & 5'-ACACTGCCATGCAAAGCATC-3' \\
Caspase-1 & 5'-ATGAATCACCAACACCAG-3' & 5'-CTTGACGCATCCTAATCC-3' \\
$\beta$-actin & 5'-CCCATCTATGAGGGTTACGC-3' & 5'-TTTAATGTCACGCACGATTTC-3'
\end{tabular}

NLRP3, NOD-like receptor family, pyrin domain-containing 3; ASC, apoptosis-associated speck-like protein containing a caspase recruitment domain (CARD).

blots were detected using the ECL-chemiluminescent kit (Thermo Scientific).

RNA interference. siRNAs targeting NLRP3 were obtained from GenePharma Corp. and contained the following sequences: NLRP3 siRNA (sense, 5'-GGCGAGACCUCUGGGAAA ATT-3' and antisense, 5'-UUUUCCCAGAGGUCUCGCCTT-3'); negative control (sense, 5'-UUCUCCGAACGUGUCACGUTT and antisense, 5'-ACGUGACACGUUCGGAG AATT-3'). The RAW264.7 (2x10\% $/ \mathrm{ml}$ cells) were cultured in 6-well plates with DMEM containing $10 \%$ FBS for $24 \mathrm{~h}$ and maintained in a $37^{\circ} \mathrm{C}$ incubator under a humidified atmosphere containing $5 \% \mathrm{CO}_{2}$ (until the density of the transfected cells reached 30 to $80 \%)$. The transfection mixture was prepared using $5 \mu \mathrm{l}$ of Lipofectamine 2000 and $4.2 \mu \mathrm{l}$ of NLRP3 siRNA, to $300 \mu \mathrm{l}$ of Opti-MEM. The siRNAs were then transfected into the RAW264.7 cells using Lipofectamine 2000 according to the instructions of the manufacturer. Knockdown efficiency was determined by qRT-PCR and western blot analysis. Following transfection, the cells were incubated for $6 \mathrm{~h}$ with antibioticsfree Opti-MEM, and were then subjected to LPS/ATP stimulation with DMEM containing 10\% FBS and used for the assays. Three independent transfection experiments were performed.

Statistical analysis. Statistic analysis was performed using SPSS 10.01 for Windows (SPSS, Inc., Chicago, IL, USA). P-values were assessed using unpaired, two-tailed Student's t-tests or one-way analysis of variance (ANOVA) with the Tukey-Kramer method. Values are reported as the means \pm standard deviation (SD). Data shown are representative of at least triplicate experiments. A P-value $<0.05$ was considered to indicate a statistically significant difference.

\section{Results}

LPS and ATP induce NLRP3 inflammasome activation, exerting a synergistic effect. Treatment with LPS (200 ng/ml) prior to treatment with $5 \mathrm{mM}$ ATP induced the upregulation of each component of the NLRP3 inflammasome in the RAW264.7 murine macrophages. The expression of NLRP3 and ASC in the RAW264.7 cells treated with LPS alone was higher than that in those stimulated with ATP alone, as shown by western blot analysis (Figs. 1 and 4). On the contrary, the expression of caspase-1 was further induced by ATP alone compared to stimulation with LPS alone (Figs. 1 and 4). Nevertheless, at the same time, dual stimulation with LPS and ATP, had a syner- gistic effect on the expression of the NLRP3 inflammasome (Figs. 1 and 4). Moreover, the mRNA levels of NLRP3, ASC and caspase-1 in the RAW264.7 cells, were further detected by qRT-PCR assay. The results revealed that the expression of NLRP3 and caspase-1 was significantly enhanced in the LPS + ATP group $(\mathrm{P}=0.003$ and $\mathrm{P}=0.002$ for NLRP3 and caspase-1, respectively) (Fig. 3), whereas the ASC levels were modestly upregulated $(\mathrm{P}=0.040)$. However, treatment with ATP had no effect on the mRNA expression of ASC compared with the control group ( $\mathrm{P}=0.140)$ (Fig. 3). Collectively, our findings showed that the mRNA and protein expression levels of the NLRP3 inflammasome were similar (Fig. 4).

High concentration of extracellular $\mathrm{K}^{+} /$knockdown of the NLRP3 gene suppresses the activation of NLRP3 and caspase-1 induced by LPS and/or ATP, but has no effect on ASC. The RAW264.7 cells were cultivated in a $150 \mathrm{nM}$ potassium environment for $30 \mathrm{~min}$ prior to stimulation with LPS and/or ATP. The protein expression of NLRP3 was moderately reduced compared with the other corresponding groups $(\mathrm{P}<0.05)$ (Fig. 1B). However, the RAW264.7 cells cultured in a high potassium milieu only demonstrated no difference compared with the control group as regards the NLRP3 level $(\mathrm{P}=0.064)$ (Fig. 1B). The ASC levels slightly decreased following stimulation with LPS + ATP in high $\mathrm{K}^{+}$cell culture medium $(\mathrm{P}=0.033)$ (Fig. 1B). Nevertheless, the expression of caspase-1 significantly decreased in the LPS group, ATP group and the LPS + ATP group $(\mathrm{P}=0.004, \mathrm{P}=0.003, \mathrm{P}=0.001$, respectively) (Fig. 1B). The expression of caspase-1 was low even without any stimuli when the RAW264.7 cells were cultured in high $\mathrm{K}^{+}$ culture medium compared with the control $(\mathrm{P}=0.023)$ (Fig. 1B). Using specific siRNA to knockdown the NLRP3 gene, we found that the expression of NLRP3 and caspase-1 significantly decreased, while the ASC levels increased compared with the other corresponding groups, as shown by qRT-PCR (Fig. 3). The results of western blot analysis for the protein expression of the NLRP3 inflammasome were similar to those obtained by qRT-PCR (Fig. 4). Of note, the decrease in the ASC levels was not statistically significant with the increasing extracellular $\mathrm{K}^{+}$ion concentration in the culture mediem (Fig. 1); NLRP3 siRNA had no effect on ASC at the mRNA and protein level. The underlying mechanisms remain unclear, and require further investigation.

ATP enhances the LPS-induced expression of $I L-1 \beta, I L-18$ and IL-33 in RAW264.7 cells. The secretion of IL-1 $\beta$ and IL-18 
A

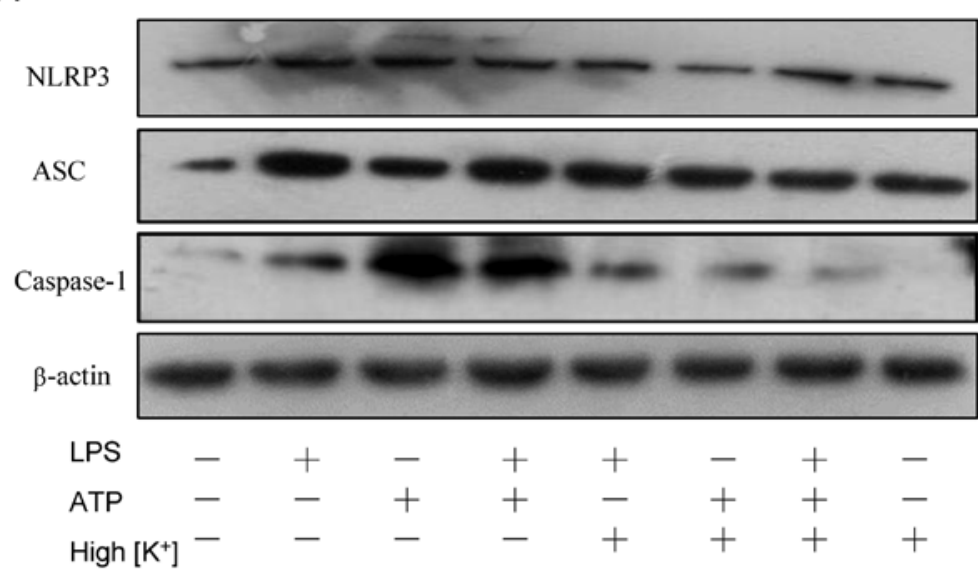

B
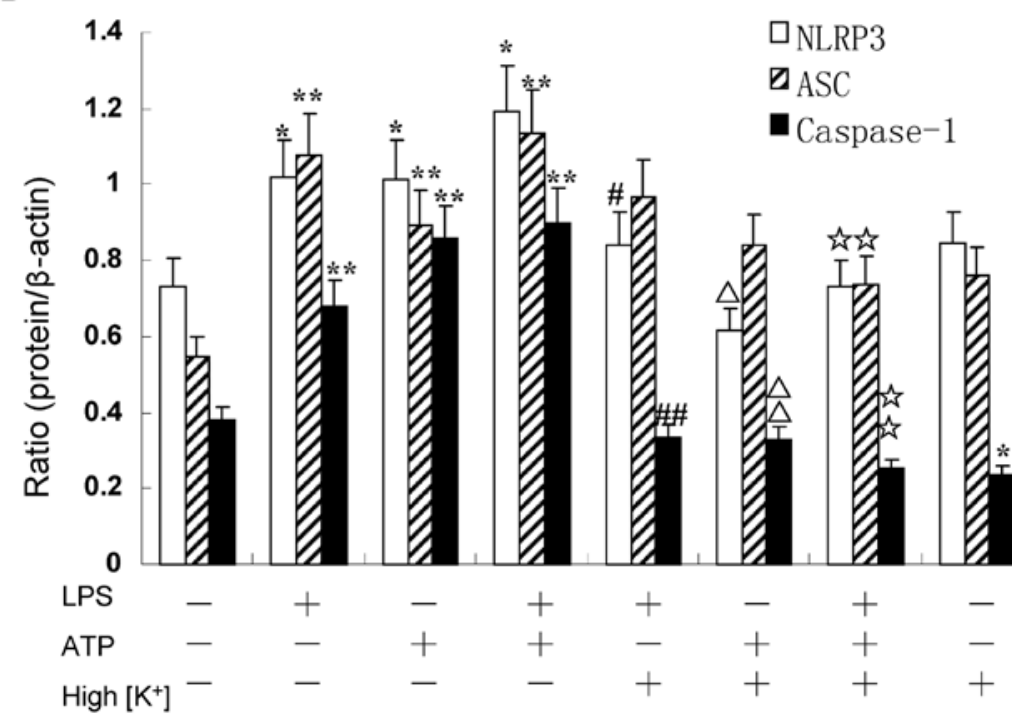

Figure 1. Treatment of RAW264.7 cells with adenosine triphosphate (ATP) and lipopolysaccharide (LPS) with or without a high concentration of extracellular potassium $\left(\mathrm{K}^{+}\right)$induced the expression of respective components of the NOD-like receptor family, pyrin domain-containing 3 (NLRP3) inflammasome (western blot analysis). RAW264.7 cells were stimulated with LPS (200 ng/ml) for $6 \mathrm{~h}$, and $2 \mathrm{mM}$ ATP for additional $30 \mathrm{~min}$ (LPS + ATP) or ATP alone. Potassium chloride $(150 \mathrm{mM})$ was additionally added to the cell culture for $30 \mathrm{~min}$ (to produce a high extracellular potassium ion environment) prior to LPS and/or ATP stimulation in the last 4 subgroups. (A) Western blot analysis of NLRP3, ASC, caspase-1 and $\beta$-actin expression in the different groups; (B) Denstitometric ratio analyses (NLRP3, ASC, caspase-1/ $\beta$-actin) using Image J software. ${ }^{*}$ Compared to control $\left({ }^{*} \mathrm{P}<0.05\right.$ and $\left.{ }^{* *} \mathrm{P}<0.01\right)$; ${ }^{*}$ compared to LPS alone $\left({ }^{\#} \mathrm{P}<0.05\right.$ and $\left.{ }^{\# \#} \mathrm{P}<0.01\right)$; ${ }^{\star}$ com-

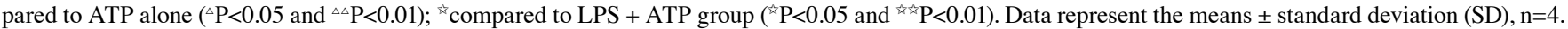

requires the induction of pro-IL-1 $\beta$ and pro-IL-18 expression, followed by its proteolytic processing to mature IL-1 $\beta$ and IL-18 by the NLRP3 inflammasome (17). The processing and generation of IL-33 are not yet clear. Caspase-1 plays a proteolytic role among the NLRP3 inflammasome complex. The RAW264.7 cells were treated with $5 \mathrm{mM}$ ATP for an additional $30 \mathrm{~min}$ in the presence or absence of $200 \mathrm{ng} / \mathrm{ml}$ LPS for $6 \mathrm{~h}$. The results revealed that the expression of IL-1 $\beta$, IL-18 and IL-33 increased when the cells were treated with a combination of LPS and ATP $(0.75 \pm 0.08,0.88 \pm 0.09$ and $0.93 \pm 0.09$, respectively; Fig. 2$)$; $(0.85 \pm 0.09,0.91 \pm 0.11$ and $0.87 \pm 0.08 \mathrm{ng} / \mathrm{ml}$, respectively; Fig. 5).

High concengtration of extracellular $K^{+} / N L R P 3$ siRNA attenuate the secretion of $I L-1 \beta$ and $I L-18$ induced by $L P S$ and/or ATP, but have no effect on IL-33. Potassium efflux is essential for the LPS and/or ATP-induced NLRP3 inflamma- some activation (18). Preventing $\mathrm{K}^{+}$efflux inhibits NLRP3 inflammasome assembly, mainly by elevating extracellular the $\mathrm{K}^{+}$concentration (19). In the present study, RAW264.7 cells were treated with a high concentration of $\mathrm{K}^{+}(150 \mathrm{nM})$ prior to any stimulation for $30 \mathrm{~min}$. The expression of IL-1 $\beta$ and IL-18 induced by LPS/ATP decreased significantly following treatment with $\mathrm{K}^{+}$, as shown by ELISA $(\mathrm{P}<0.01)$ (Fig. $2 \mathrm{~A}$ and $\mathrm{B}$ ). It is noteworthy that the secretion of IL-18 still decreased without any external stimuli when the RAW264.7 cells were cultured in high $\mathrm{K}^{+}$culture medium compared with the control ( $\mathrm{P}=0.034)$ (Fig. 2B). However, a high concentration of $\mathrm{K}^{+}(150 \mathrm{mM})$ blocked the expression and release of IL-1 $\beta$ and IL-18, but not that of IL-33 ( $\mathrm{P}>0.05$ ) (Fig. 2C). The low protein expression of NLRP3 may abrogate NLRP3 inflammasome assembly. Thus, similar results were observed on these 3 cytokines when the NLRP3 gene was knocked down using NLRP3 siRNA (Fig. 5). Specific siRNAs were used to 


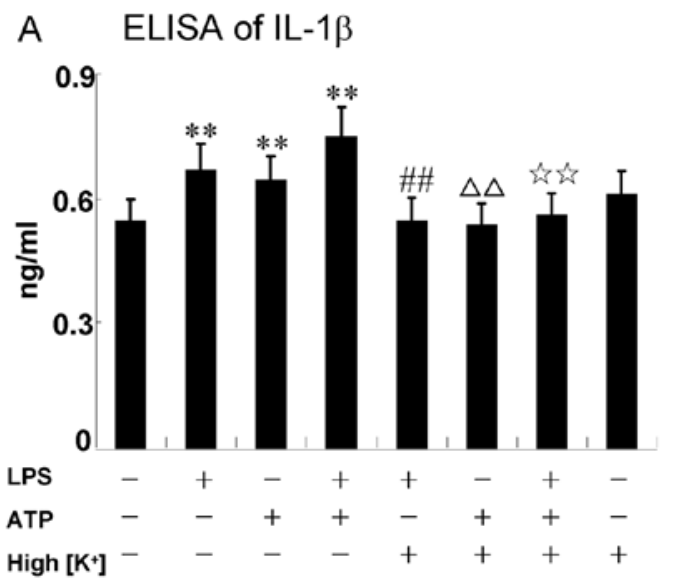

B ELISA of IL-18

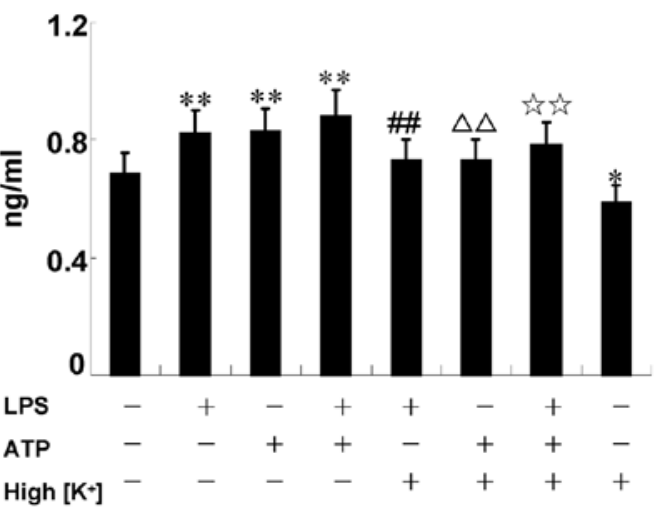

\section{ELISA of IL-33}

\section{2}

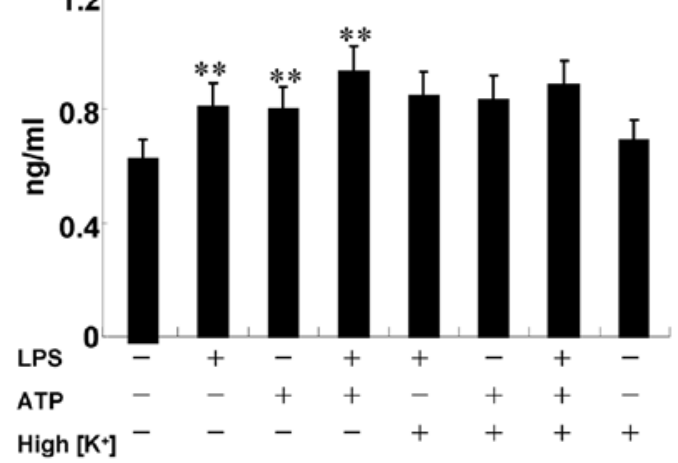

Figure 2. Treatment of RAW264.7 cells with adenosine triphosphate (ATP) and lipopolysaccharide (LPS) with or without a high concentration of extracellular potassium $\left(\mathrm{K}^{+}\right)$induced the secretion of cytokines, including interleukin (IL)-1 $\beta$, IL-18 and IL-33, as shown by enzyme-linked immunosorbent assay (ELISA) . RAW264.7 cells were stimulated with LPS $(200 \mathrm{ng} / \mathrm{ml})$ for $6 \mathrm{~h}$, and $2 \mathrm{mM}$ ATP for an additional $30 \mathrm{~min}$ (LPS + ATP) or ATP alone. Potassium chloride $(150 \mathrm{mM})$ was additionally added to the cell culture for $30 \mathrm{~min}$ (to produce a high extracellular potassium ion environment) prior to LPS and/or ATP stimulation in the last 4 subgroups. Following treatment, the supernatants were examined. (A-C) Expression of IL-1 $\beta$, IL-18 and IL-33 in the supernatants of different groups, as shown by ELISA. ${ }^{*}$ Compared to control $\left({ }^{*} \mathrm{P}<0.05\right.$ and $\left.{ }^{* *} \mathrm{P}<0.01\right) ;{ }^{*}$ compared to LPS alone $\left({ }^{\# \#} \mathrm{P}<0.01\right) ;{ }^{\wedge}$ compared to ATP alone $\left({ }^{\triangle} \wedge \mathrm{P}<0.01\right) ;{ }^{*}$ compared to LPS + ATP group ( $\left.{ }^{\text {(ix }} \mathrm{P}<0.01\right)$. Data represent the means \pm standard deviation $(\mathrm{SD}), \mathrm{n}=4$.

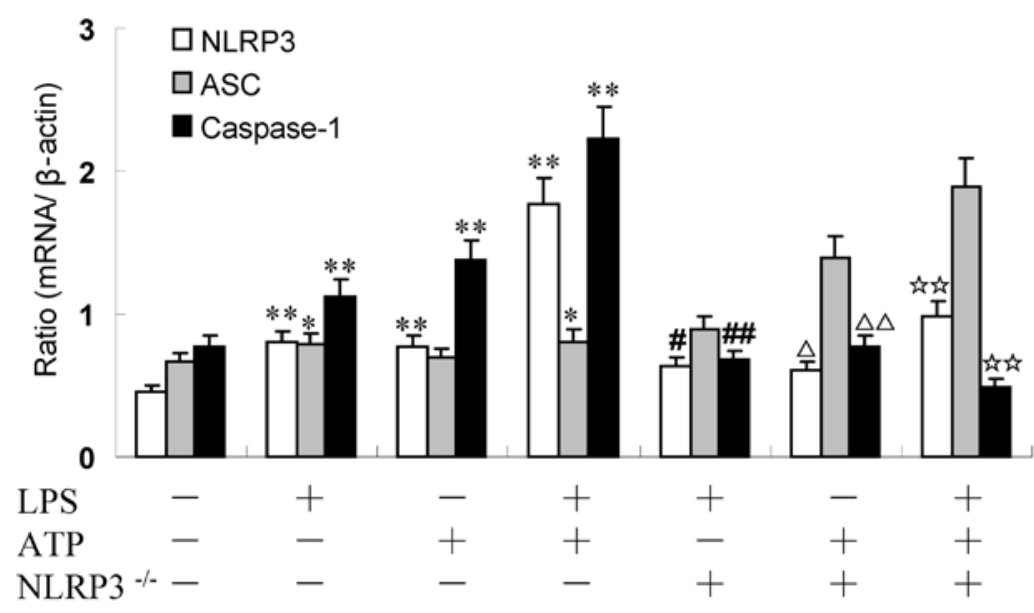

Figure 3. mRNA expression of NOD-like receptor family, pyrin domain-containing 3 (NLRP3), ASC, and caspase-1 in RAW264.7 cells treated with lipopolysaccharide (LPS) $(200 \mathrm{ng} / \mathrm{ml})$, adenosine triphosphate (ATP) ( $2 \mathrm{mM})$ with or without NLRP3 small interfering (siRNA), as shown by qRT-PCR. "Compared to the control $\left({ }^{*} \mathrm{P}<0.05\right.$ and $\left.{ }^{* *} \mathrm{P}<0.01\right) ;{ }^{*}$ compared to LPS alone $\left({ }^{*} \mathrm{P}<0.05\right.$ and $\left.{ }^{\# \#} \mathrm{P}<0.01\right) ;{ }^{*}$ compared to ATP alone $\left({ }^{\triangle} \mathrm{P}<0.05\right.$ and $\left.{ }^{\triangle \Delta} \mathrm{P}<0.01\right) ;{ }^{* *}$ compared to LPS $+\mathrm{ATP}$ group $\left({ }^{*} \mathrm{P}<0.05\right.$ and $\left.{ }^{\text {thi }} \mathrm{P}<0.01\right)$ Data represent the means \pm standard deviation $(\mathrm{SD}), \mathrm{n}=4$.

knock down the NLRP3 gene in order to investigate the effects on the expression and release of cytokines in RAW264.7 cells (Fig. 5). The results revealed that the NLRP3 knockdown decreased IL-18 expression ( $\mathrm{P}<0.01)$ (Fig. 5B), and a certain decrease in IL-1 $\beta$ expression was also observed $(\mathrm{P}<0.05)$ (Fig. 5A). We also found a non-significant, but minor increase 
A

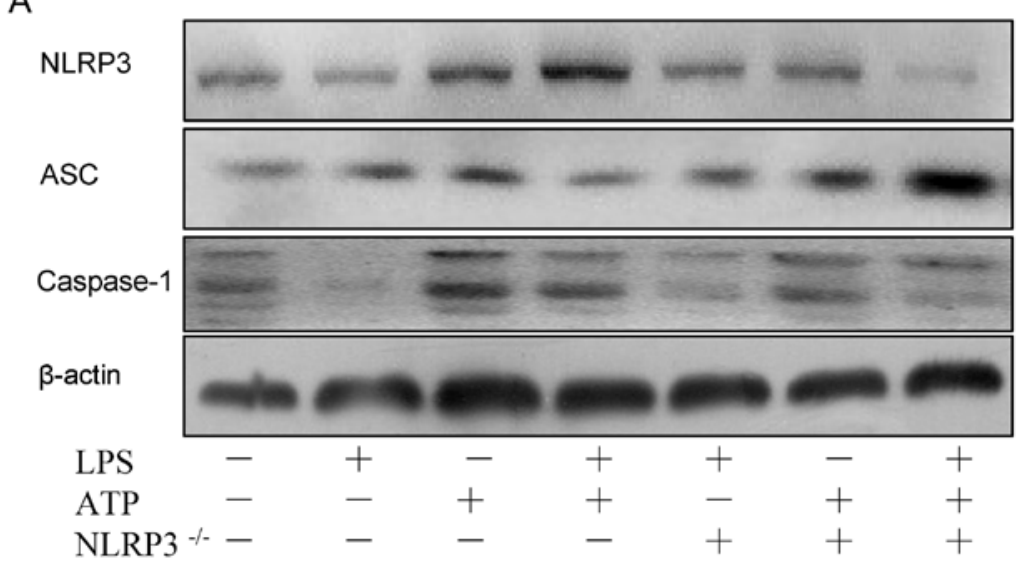

B

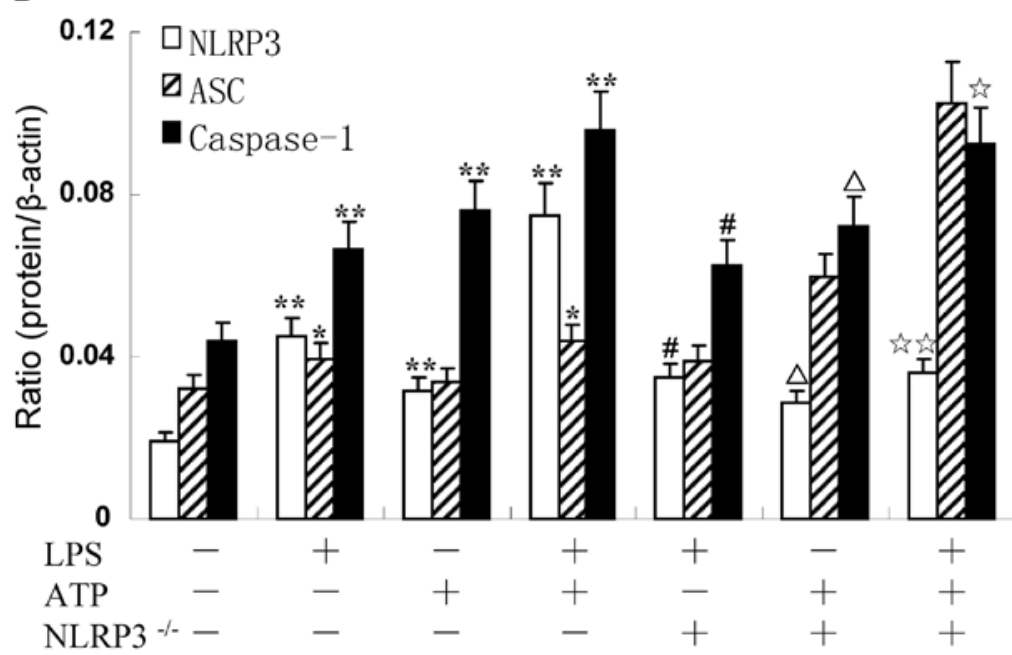

Figure 4. Treatment of RAW264.7 cells with adenosine triphosphate (ATP) and lipopolysaccharide (LPS) with or without NOD-like receptor family, pyrin domain-containing 3 (NLRP3) small interfering (siRNA) induced the expression of respective components of NLRP3 inflammasome, as shown by western blot analysis. RAW264.7 cells were stimulated with LPS $(200 \mathrm{ng} / \mathrm{ml})$ for $6 \mathrm{~h}$ and $2 \mathrm{mM}$ ATP for an additional $30 \mathrm{~min}$ (LPS + ATP) or ATP alone. We transfected RAW264.7 cells with NLRP3 siRNA prior to stimulation with LPS and/or ATP in the last 3 subgroups. (A) Western blot analysis of NLRP3, ASC, caspase-1 and $\beta$-actin in the different groups; (B) Denstitometric ratio analyses (NLRP3, ASC, caspase-1/ $\beta$-actin) using ImageJ software. "Compared to control

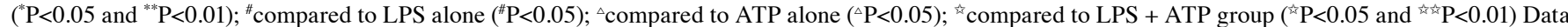
represent the means \pm standard deviation $(\mathrm{SD}), \mathrm{n}=4$.

in IL-33 expression after the knockdown of NLRP3, compared to the ATP alone and LPS + ATP group ( $\mathrm{P}>0.05)$ (Fig. 5C). However, the IL-33 levels were decreased compared with the LPS group ( $\mathrm{P}=0.037$ ) (Fig. 5C).

\section{Discussion}

The significance of innate immunity lies not only in comprising the first line of defense against pathogenic and non-pathogenic insults, but also in developing an efficient adaptive immune response (20-24). The NLRP3 inflammasome provides multiprotein molecular platforms for the activation of caspase-1 and subsequent processing and the secretion of IL-1 family members, to engage innate immune defense $(5,24)$. Given the importance of IL-1 family members associated with acute and chronic inflammation, understanding the role of the NLRP3 inflammasome in the initiation of innate immune response cannot be ignored (24). Therefore, we examined the effects of LPS/ATP on the expression of inflammatory cytokines and the components of the NLRP3 inflammasome in RAW264.7 macrophage cells. There are two signals required for the activation of the NLRP3 inflammasome in macrophages, the priming of the cells triggered by LPS, and extracellular ATP, which enables the subsequent orchestration of a robust processing and the release of inflammatory cytokines, such as IL-1 $\beta$ and IL-18 (Fig. 6) (25). In our study, we found that LPS and/or ATP stimulation induced the activation of the NLRP3 inflammasome and the sequential use of LPS and ATP exerted synergistic effects on the protein expression of the NLRP3 inflammasome (Figs. 1 and 4).

Understanding the molecular mechanisms of NLRP3 inflammasome activation has become a focus or research. Mariathasan et al (11) found that the NLRP3 inflammasome can be activated by extracellular ATP stimulation. In addition, hyaluronan and $\beta$-amyloid plaque, the causative agents of Alzheimer's disease, elevate plasma glucose, as well as a diversity of environmental insults, including silica, have been found to induce NLRP3 inflammasome activation (26-32). Similarly, exposure to UVB irradiation can trigger NLRP3 inflammasome activation (33). TLR4 can bind to LPS directly (one of 
A ELISA of IL-1 $\beta$

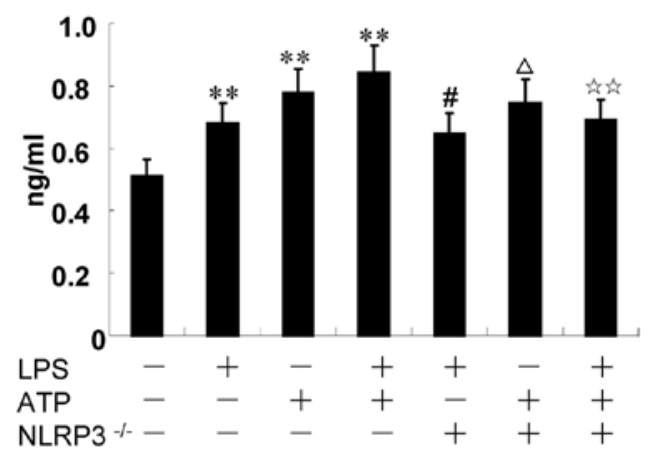

B ELISA of IL-18

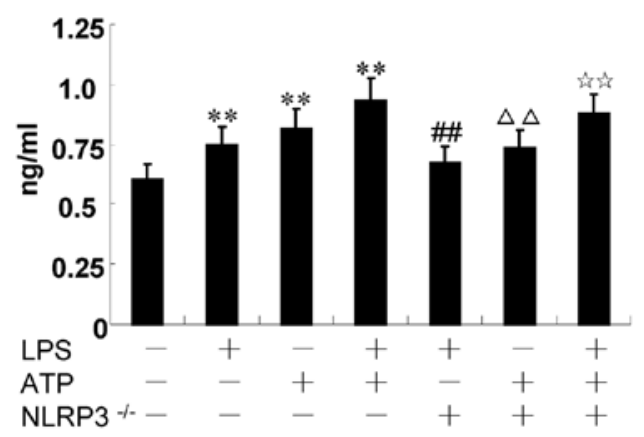

\section{ELISA of IL-33}

1.25

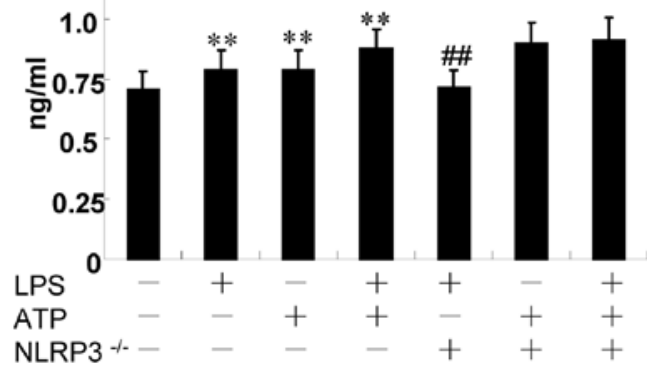

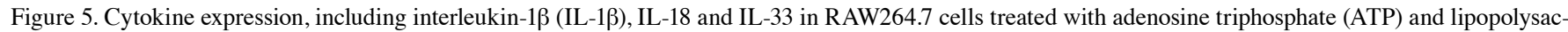
charide (LPS) with or without NOD-like receptor family, pyrin domain-containing 3 (NLRP3) small interfering (siRNA), as shown by ELISA. RAW264.7 cells were stimulated with LPS $(200 \mathrm{ng} / \mathrm{ml})$ for $6 \mathrm{~h}$, and $2 \mathrm{mM}$ ATP for an additional $30 \mathrm{~min}$ (LPS + ATP) or ATP alone. We transfected the RAW264.7 cells with NLRP3 siRNA prior to stimulation with LPS and/or ATP in the last 3 subgroups. Following treatment, the supernatants were examined. (A-C) Expression of IL-1 $\beta$, IL-18 and IL-33 in supernatants of the different groups. "Compared to control $\left({ }^{* *} \mathrm{P}<0.01\right) ;{ }^{*}$ compared to LPS alone $\left({ }^{\#} \mathrm{P}<0.05\right.$ and $\left.{ }^{\# \#} \mathrm{P}<0.01\right) ;{ }^{\circ}$ compared to ATP alone $\left({ }^{\triangle} \mathrm{P}<0.05\right.$ and $\left.{ }^{\triangle} \mathrm{P}<0.01\right) ;{ }^{\wedge}$ compared to LPS + ATP group $\left({ }^{\wedge} \vec{\wedge} \mathrm{P}<0.01\right)$. Data represent the means \pm standard deviation $(\mathrm{SD}), \mathrm{n}=4$.

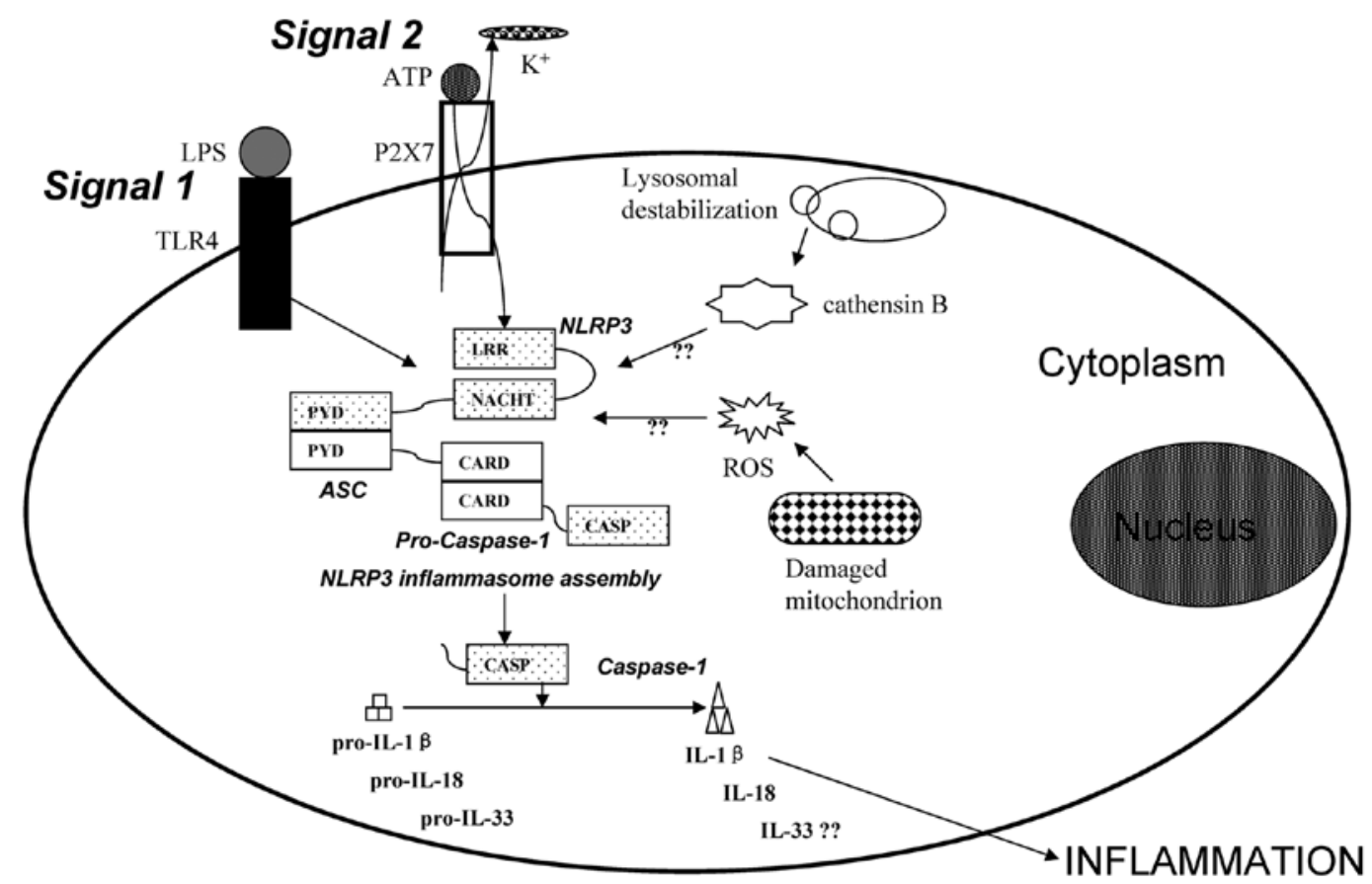

Figure 6. Suggested model of the activation of NOD-like receptor family, pyrin domain-containing 3 (NLRP3) inflammasome including 3 models: potassium (K+) efflux, reactive oxygen species (ROS) generation and lysosomal destabilization.

the PAMPs), and ATP (one of the DAMPs) can bind to P2X7R (one of the purine receptors). However, given the divergent structures of DAMPs and PAMPs, it is likely that these agonists cannot bind to NLRP3 directly. Thus, the mechanisms through 
which PAMPs and DAMPs activate the NLRP3 inflammasome remain ambiguous. These mechanisms have thus far proved to be elusive $(34,35)$. However, current opinion suggests that 3 models, including $\mathrm{K}^{+}$efflux, lysosomal destabilization and ROS production, can activate the signaling pathway that results in NLRP3 inflammasome activation (Fig. 6) (36). Extracellular ATP connects with the ATP-gated cation channel P2X7R when cells are under attack by various insults, and then the connection of ligand and receptor can cause membrane pore formation to trigger $\mathrm{K}^{+}$efflux. Thus, suppressing $\mathrm{K}^{+}$efflux can inhibit the expression of the NLRP3 inflammasome. In the present study, $150 \mathrm{mM} \mathrm{K}^{+}$(much more than the physiological concentration of $\mathrm{K}^{+}$inside the cell) was used to suppress $\mathrm{K}^{+}$efflux. Our findings revealed that the expression of NLRP3 $(\mathrm{P}<0.05)$ and caspase-1 $(\mathrm{P}<0.01)$ were suppressed by the increased concentration of extracellular $\mathrm{K}^{+}$(Fig. 1). It has been known that transfecting osteoblasts with siRNA can knock down NLRP3 expression (37). Accordingly, the present study found that the mRNA and protein levels of NLRP3 and caspase-1 decreased following the transfection of NLRP3 siRNA into RAW264.7 cells (Figs. 3 and 4). NLRP3 can undergo self-oligomerization in the processing of PAMP or DAMP recognition, and then recruits ASC, which is the crucial adaptor protein by PYD-PYD domain interaction (Fig. 6) (38,39). ASC subsequently bridges to caspase-1 through CARD-CARD interaction (Fig. 6) $(40,41)$. Prior to NLRP3 inflammasome activation, ASC is located in the nucleus of cells completely in order to prevent caspase-1 activation and the subsequent processing and generation of cytokines, such as IL-1 $\beta$ and IL-18 (42). Once NLRP3 molecular platforms are activated by PAMPs and DAPMs, ASC is redistributed from the nucleus to the cytosol, where it aggregates with NLRP3 and caspase- 1 and then transforms into perinuclear structures to achieve the assembly of the NLRP3 inflammasome (43). Available evidence suggests that the redistribution of ASC may function as a checkpoint to prevent spontaneous and harmful inflammasome activation (42). Furthermore, the quantity of the protein expression of ASC and NLRP3 is not in parallel (42). In agreement with this viewpoint, our study indicated that a high concontration of extracellular $\left[\mathrm{K}^{+}\right] / \mathrm{NLRP} 3$ siRNA downregulated the expression of NLRP3 and caspase-1 induced by LPS and/or ATP, but had little effect on ASC (Figs. 1, 3 and 4). Nevertheless, the precise mechanisms that regulate inflammasomes at the level of ASC require further clarification.

Caspase-1 is the first identified caspase, which is generally presented in the cytosol of phagocytic cells as pro-caspase-1 in the form of inactive zymogen (44). Following stimulation with various microbial or various endogenous signals, pro-caspase-1 is self-cleavaged by proteolytic cleavage from zymogen into the enzymatically active heterodimer, composed of two 10 and $20 \mathrm{kDa}$ subunits (45). Active caspase-1 is essential for the cleavage of pro-IL-1 $\beta$ and pro-IL-18 into their biologically active mature forms. Mature IL- $1 \beta$ is involved in many immune reactions, including the recruitment of inflammatory cells to the site of infection, whereas IL-18 plays an essential role in the production of interferon- $\gamma($ IFN- $\gamma$ ) and enhancement of the cytolytic activity of natural killer (NK) cells (46). When the expression of caspase-1 is upregulated or downregulated, the secretion of IL-1 $\beta$ and IL-18 will increase or decrease correspondingly. Our data indicated that LPS and ATP stimulation induced the activation of caspase-1 (Figs. 1 and 4), and the robust release of
IL-1 $\beta$ and IL-18 (Figs. 2 and 5). However, the decreased levels of caspase-1 (Figs. 1 and 4) accompanied with the reduction of IL-1 $\beta$ and IL-18 (Figs. 2 and 5) were detected in both the extracellular high $\mathrm{K}^{+}$milieu- and NLRP3 siRNA-treated groups. IL-33 belongs to the IL-1 subfamily (12), is closely related to IL-1 $\beta$ and IL-18 as there is a caspase- 1 site in the structure of the IL-33 precursor (47). The construction of IL-33 is closer to IL-18 than IL-1 $\beta$. However, evidence suggests that the caspase-1 cleavage site is similar to the consensus sequence for caspase-3 and that the intracellular IL-33 precursor is also a substrate for caspase-3 (48). Neutrophil proteinase 3 (PR3) can process the precursor IL-33 into an active mature form as IL-33, but increasing PR3 incubation time downregulates the biological activity of IL-33 (49). In addition, neutrophil elastase and cathepsin G can cleave the IL-33 precursor, which results in the maturation of precursor IL-33 (50). Thus, extracellular IL-33 is secreted as a precursor and can be processed by a diversity of enzymes apart from caspase-1. Given the complexity of IL-33 maturity processing, our data indicated that the expression of IL-33 ( $\mathrm{P}>0.05)$ did not decrease with the downregulation of caspase -1 by the addition of a high concentration of $\mathrm{K}^{+}$into the cell culture medium or by using siRNA to knock down the NLRP3 gene (Figs. 2 and 5). This may elucidate the underlying mechanisms of the processing of precursor IL-33 for a variety of hydrolytic enzymes participating in this process.

Autoimmune disease pathogenesis is related to cytokines, such as IL-1 $\beta$ and IL-18 and anakinra has been used to block IL-1 $\beta$ activity to treat rheumatoid arthritis (RA) (51). However, the single use of anakinra is less effective as other anticytokine therapies are more effective (51). Thus, it is possible that the inhibition of the NLRP3 inflammasome could provide a therapeutic target than specific IL-1 blockade, whereby blocking the NLRP3 inflammasome could block IL-1 $\beta$ and IL-18 activity simultaneously, both of which play critical roles in the progression of RA. Nevertheless, further studies are required to confirm this hypothesis.

Collectively, in the present study, LPS/ATP was utilized to induce the activation of the NLRP3 inflammasome. A high concentration of extracellular $\mathrm{K}^{+}$and the knockdown of NLRP3 using siRNA can block the secretion of IL-1 $\beta$ and IL-18 in RAW264.7 cells.

\section{Acknowledgements}

Our study was supported by grants from the Anhui Science and Technology Tackling Fund (no. 1206c0805026), and the National Science Foundation of China (nos. 81072685 and 81273526).

\section{References}

1. Martinon F, Mayor A and Tschopp J: The inflammasomes: guardians of the body. Annu Rev Immunol 27: 229-265, 2009.

2. Franchi L, Eigenbrod T and Núñez G: Cutting edge: TNF-alpha mediates sensitization to ATP and silica via the NLRP3 inflammasome in the absence of microbial stimulation. J Immunol 183: 792-796, 2009.

3. Jin C and Flavell RA: Molecular mechanism of NLRP3 inflammasome activation. J Clin Immunol 30: 628-631, 2010.

4. Conforti-Andreoni C, Ricciardi-Castagnoli P and Mortellaro A: The inflammasomes in health and disease: from genetics to molecular mechanisms of autoinflammation and beyond. Cell Mol Immunol 8: 135-145, 2011. 
5. Schroder K and Tschopp J: The inflammasomes. Cell 140 821-832, 2010

6. Chen M, Wang H, Chen W and Meng G: Regulation of adaptive immunity by the NLRP3 inflammasome. Int Immunopharmacol 11: 549-554, 2011.

7. Leemans JC, Cassel SL and Sutterwala FS: Sensing damage by the NLRP3 inflammasome. Immunol Rev 243: 152-162, 2011.

8. Davis BK, Wen H and Ting JP: The inflammasome NLRs in immunity, inflammation, and associated diseases. Annu Rev Immunol 29: 707-735, 2011.

9. Cassel SL, Joly S and Sutterwala FS: The NLRP3 inflammasome: a sensor of immune danger signals. Semin Immunol 21: 194-198, 2009.

10. Hsu HY and Wen MH: Lipopolysaccharide-mediated reactive oxygen species and signal transduction in the regulation of interleukin-1 gene expression. J Biol Chem 277: 22131-22139, 2002.

11. Mariathasan S, Weiss DS, Newton K, McBride J, O'Rourke K, Roose-Girma M, Lee WP, Weinrauch Y, Monack DM and Dixit VM: Cryopyrin activates the inflammasome in response to toxins and ATP. Nature 440: 228-232, 2006

12. van de Veerdonk FL and Netea MG: New insights in the immunobiology of IL-1 family members. Front Immunol 4: 167, 2013.

13. Qi J, Ye X, Ren G, Kan F, Zhang Y, Guo M, Zhang Z and Li D: Pharmacological efficacy of anti-IL-1 $\beta$ scFv, Fab and full-length antibodies in treatment of rheumatoid arthritis. Mol Immunol 57: 59-65, 2014.

14. Voronov E, Dayan M, Zinger H, Gayvoronsky L, Lin JP, Iwakura Y, Apte RN and Mozes E: IL-1 beta-deficient mice are resistant to induction of experimental SLE. Eur Cytokine Netw 17: 109-116, 2006.

15. Girardin SE: Knocking in the NLRP3 inflammasome. Immunity 30: 761-763, 2009.

16. Latz E: The inflammasomes: mechanisms of activation and function. Curr Opin Immunol 22: 28-33, 2010.

17. Budai MM, Varga A, Milesz S, Tozser J and Benko S: Aloe vera downregulates LPS-induced inflammatory cytokine production and expression of NLRP3 inflammasome in human macrophages. Mol Immunol 56: 471-479, 2013.

18. Hussen J, Düvel A, Koy M and Schuberth HJ: Inflammasome activation in bovine monocytes by extracellular ATP does not require the purinergic receptor P2X7. Dev Comp Immunol 38 : 312-320, 2012

19. Pétrilli V, Papin S, Dostert C, Mayor A, Martinon F and Tschopp J: Activation of the NALP3 inflammasome is triggered by low intracellular potassium concentration. Cell Death Differ 14: 1583-1589, 2007.

20. Kawai $\mathrm{T}$ and Akira S: Innate immune recognition of viral infection. Nat Immunol 7: 131-137, 2006.

21. Bose $S$ and Banerjee $A K$ : Innate immune response against nonsegmented negative strand RNA viruses. J Interferon Cytokine Res 23: 401-412, 2003

22. O'Neill LA and Bowie AG: Sensing and signaling in antiviral innate immunity. Curr Biol 20: R328-R333, 2010.

23. Rathinam VA and Fitzgerald KA: Inflammasomes and anti-viral immunity. J Clin Immunol 30: 632-637, 2010.

24. Ciraci C, Janczy JR, Sutterwala FS and Cassel SL: Control of innate and adaptive immunity by the inflammasome. Microbes Infect 14: 1263-1270, 2012

25. Mankan AK, Dau T, Jenne D and Hornung V: The NLRP3/ASC/ Caspase-1 axis regulates IL-1beta processing in neutrophils. Eur J Immunol 42: 710-715, 2012

26. Yamasaki K, Muto J, Taylor KR, Cogen AL, Audish D, Bertin J, Grant EP, Coyle AJ, Misaghi A, Hoffman HM and Gallo RL: NLRP3/cryopyrin is necessary for interleukin-1beta (IL-1beta) release in response to hyaluronan, an endogenous trigger of inflammation in response to injury. J Biol Chem 284: 12762-12771, 2009.

27. Halle A, Hornung V, Petzold GC, Stewart CR, Monks BG, Reinheckel T, Fitzgerald KA,LatzE, Moore KJ and Golenbock DT: The NALP3 inflammasome is involved in the innate immune response to amyloid-beta. Nat Immunol 9: 857-865, 2008

28. Zhou R, Tardivel A, Thorens B, Choi I and Tschopp J: Thioredoxin-interacting protein links oxidative stress to inflammasome activation. Nat Immunol 11: 136-140, 2010.

29. Dostert C, Pétrilli V, Van Bruggen R, Steele C, Mossman BT and Tschopp J: Innate immune activation through Nalp3 inflammasome sensing of asbestos and silica. Science 320: 674-677, 2008.

30. Hornung V, Bauernfeind F, Halle A, Samstad EO, Kono H, Rock KL, Fitzgerald KA and Latz E: Silica crystals and aluminum salts activate the NALP3 inflammasome through phagosomal destabilization. Nat Immunol 9: 847-856, 2008
31. Cassel SL, Eisenbarth SC, Iyer SS, Sadler JJ, Colegio OR, Tephly LA, Carter AB, Rothman PB, Flavell RA and Sutterwala FS: The Nalp3 inflammasome is essential for the development of silicosis. Proc Natl Acad Sci USA 105: 9035-9040, 2008.

32. Eisenbarth SC, Colegio OR, O'Connor W, Sutterwala FS and Flavell RA: Crucial role for the Nalp3 inflammasome in the immunostimulatory properties of aluminium adjuvants. Nature 453: 1122-1126, 2008

33. Feldmeyer L, Keller M, Niklaus G, Hohl D, Werner S and Beer HD: The inflammasome mediates UVB-induced activation and secretion of interleukin-1beta by keratinocytes. Curr Biol 17: $1140-1145,2007$

34. Bryant $\mathrm{C}$ and Fitzgerald KA: Molecular mechanisms involved in inflammasome activation. Trends Cell Biol 19: 455-464, 2009.

35. McGettrick AF and O'Neill LA: NLRP3 and IL-1beta in macrophages as critical regulators of metabolic diseases. Diabetes Obes Metab 15 (Suppl 3): 19-25, 2013.

36. Lamkanfi $\mathbf{M}$ and Dixit VM: Inflammasomes: guardians of cytosolic sanctity. Immunol Rev 227: 95-105, 2009.

37. McCall SH, Sahraei M, Young AB, Worley CS, Duncan JA, Ting JP and Marriott I: Osteoblasts express NLRP3, a nucleotidebinding domain and leucine-rich repeat region containing receptor implicated in bacterially induced cell death. J Bone Miner Res 23: 30-40, 2008

38. Duncan JA, Bergstralh DT, Wang Y, Willingham SB, Ye Z, Zimmermann AG and Ting JP: Cryopyrin/NALP3 binds ATP/ dATP, is an ATPase, and requires ATP binding to mediate inflammatory signaling. Proc Natl Acad Sci USA 104: 8041-8046, 2007.

39. Faustin B, Lartigue L, Bruey JM, Luciano F, Sergienko E, Bailly-Maitre B, Volkmann N, Hanein D, Rouiller I and Reed JC: Reconstituted NALP1 inflammasome reveals two-step mechanism of caspase-1 activation. Mol Cell 25: 713-724, 2007.

40. Srinivasula SM, Poyet JL, Razmara M, Datta P, Zhang Z and Alnemri ES: The PYRIN-CARD protein ASC is an activating adaptor for caspase-1. J Biol Chem 277: 21119-21122, 2002.

41. Stehlik C, Lee SH, Dorfleutner A, Stassinopoulos A, Sagara J and Reed JC: Apoptosis-associated speck-like protein containing a caspase recruitment domain is a regulator of procaspase-1 activation. J Immunol 171: 6154-6163, 2003

42. Bryan NB, Dorfleutner A, Kramer SJ, Yun C, Rojanasakul Y and Stehlik C: Differential splicing of the apoptosis-associated speck like protein containing a caspase recruitment domain (ASC) regulates inflammasomes. J Inflamm (Lond) 7: 23, 2010.

43. Bryan NB, Dorfleutner A, Rojanasakul Y and Stehlik C: Activation of inflammasomes requires intracellular redistribution of the apoptotic speck-like protein containing a caspase recruitment domain. J Immunol 182: 3173-3182, 2009.

44. Franchi L1, Eigenbrod T, Muñoz-Planillo R and Nuñez G: The inflammasome: a caspase-1-activation platform that regulates immune responses and disease pathogenesis. Nat Immunol 10: 241-247, 2009.

45. Martinon $\mathrm{F}$ and Tschopp J: Inflammatory caspases: linking an intracellular innate immune system to autoinflammatory diseases. Cell 117: 561-574, 2004

46. Arend WP, Palmer G and Gabay C: IL-1, IL-18, and IL-33 families of cytokines. Immunol Rev 223: 20-38, 2008.

47. Schmitz J, Owyang A, Oldham E, Song Y, Murphy E, McClanahan TK, Zurawski G, Moshrefi M, Qin J, Li X, Gorman DM, Bazan JF and Kastelein RA: IL-33, an interleukin1-like cytokine that signals via the IL-1 receptor-related protein ST2 and induces T helper type 2-associated cytokines. Immunity 23: 479-490, 2005.

48. Cayrol C and Girard JP: The IL-1-like cytokine IL-33 is inactivated after maturation by caspase-1. Proc Natl Acad Sci USA 106: 9021-9026, 2009.

49. Bae S, Kang T, Hong J, Lee S, Choi J, Jhun H, Kwak A, Hong K, Kim E, Jo S and Kim S: Contradictory functions (activation/ termination) of neutrophil proteinase 3 enzyme (PR3) in interleukin-33 biological activity. J Biol Chem 287: 8205-8213, 2012.

50. Lefrancais E, Roga S, Gautier V, Gonzalez-de-Peredo A, Monsarrat B, Girard JP and Cayrol C: IL-33 is processed into mature bioactive forms by neutrophil elastase and cathepsin $\mathrm{G}$. Proc Natl Acad Sci USA 109: 1673-1678, 2012

51. Shaw PJ, McDermott MF and Kanneganti TD: Inflammasomes and autoimmunity. Trends Mol Med 17: 57-64, 2011. 